\title{
The IgE Blocking Activity Induced by Dermatophagoides pteronyssinus Subcutaneous Immunotherapy Does Not Correlate with Specific IgA but with IgG4 in both Serum and Saliva
}

\author{
${\text { Yong } \mathrm{He}^{\mathrm{a}} \text { Jinling Liu }}^{\mathrm{b}}$ Deyu Zhao ${ }^{\mathrm{c}}$ Suqin Zhang ${ }^{\mathrm{d}}$ Guodong Hao $^{\mathrm{e}}$ \\ Yuemei Sun ${ }^{f}$ Haifeng Zhong ${ }^{g}$ Huandong Chen ${ }^{g}$ Peter Adler Würtzen ${ }^{h}$ \\ Jørgen Nedergaard Larsen ${ }^{i}$ Xuxin Lai ${ }^{9}$

\begin{abstract}
aDepartment of Otolaryngology Head and Neck Surgery, The Affiliated Hospital of Medical School, Ningbo University, Ningbo, China; 'bepartment of Pulmonology, The Children's Hospital, National Clinical Research Center for Child Health, Zhejiang University School of Medicine, Hangzhou, China; 'Department of Respiratory Medicine, Children's Hospital of Nanjing Medical University, Nanjing, China; ${ }^{\mathrm{d} D e p a r t m e n t}$ of Allergy, The First Affiliated Hospital of Zhengzhou University, Zhengzhou, China; 'Department of Allergy, Tangshan Gongren Hospital, Tangshan, China; fDepartment of Allergy, Yantai YuHuangDing Hospital, Yantai, China; ${ }^{9}$ Scientific affairs, ALK A/S, Guangzhou, China; ${ }^{\text {h} T r a n s l a t i o n a l ~ R e s e a r c h, ~ A L K ~ A / S, ~ H o r s h o l m, ~ D e n m a r k ; ~ ' G l o b a l ~ M e d i c a l ~ A f f a i r s, ~ A L K ~ A / S, ~}$ Horsholm, Denmark
\end{abstract}

\section{Keywords}

House dust mite $\cdot \lg \mathrm{A} \cdot \lg \mathrm{E}$ blocking activity $\cdot$ Salivary $\lg \mathrm{G} 4 \cdot$

Subcutaneous-specific immunotherapy

\begin{abstract}
Background: The role of salivary-specific $\lg G 4$ and $\lg \mathrm{A}$ in subcutaneous immunotherapy (SCIT) is not well defined. We aimed to investigate the change of $\lg G 4$ and $\lg A$ in both serum and saliva and their correlations with IgE-blocking-factor (IgE-BF) during SCIT. Method: 307 Dermatophagoides pteronyssinus (DP) allergic rhinitis and/or asthma patients were recruited for this study. 286 patients received DP-SCIT for 1 year. Twenty-one patients received only symptomatic treatment. DP-, Der p 1-, and Der p 2-specific IgE in serum, specific-lgG4 and Der p 2-specific $\lg A 1$ and $\lg A 2$ in both serum and saliva were measured at timepoints 0,4 , and 12 months during DP-SCIT. Correlation between salivary and
\end{abstract}

serological $\lg G 4, \lg A$, and their correlation with DP-specific IgE-BF measured in serum was evaluated. Results: During DP-SCIT, the allergen-specific IgG4 in both saliva and serum increased and correlated significantly, the correlation becomes stronger over the treatment time. DP-specific IgE-BF significantly correlated with DP-specific IgG4 in serum ( $p<$ $0.0001)$ at different timepoints and in saliva at 12 months of SCIT $(p<0.01)$. No change in Der $p 2$-specific IgA during DPSCIT was observed, and the $\lg A$ in serum did not correlate with $\lg A$ in saliva. There was no correlation between DP IgEBF and Der $p$ 2-specific IgA in serum or saliva. The control group did not exhibit significant changes in any antibody level measured. Conclusion: The IgE blocking activity induced by DP-SCIT treatment correlated with specific IgG4

Yong He, Jinling Liu, Deyu Zhao, Suqin Zhang, Guodong Hao, and Yuemei Sun contributed equally.

Edited by: H.-U. Simon, Bern.
Correspondence to:

Xuxin Lai, xuxin.lai@alk.net 
and not $\lg A$. The $\lg G 4$ in saliva correlates with serum $\lg G 4$ and can be an alternative immunological marker beyond 1 year of SCIT treatment.

(c) 2021 S. Karger AG, Basel

\section{Introduction}

Clinical and immunological tolerance is the symbols of successful allergen immunotherapy (AIT). The clinical benefit of AIT includes symptom improvement, reduction in medication use, and improved quality of life (QoL). The immune tolerance of AIT is associated with suppression of allergic inflammatory responses, induction of regulatory $\mathrm{T}$ cells, and restoration of the balance between allergen-specific Th1/Th2 responses, in addition to induction of IgE blocking antibodies $[1,2]$.

Subcutaneous immunotherapy (SCIT) using standardized allergen vaccine is a classical causal treatment for type 1 allergy. House dust mite (HDM) is the most important cause of allergy in China [3], and HDM Dermatophagoides pteronyssinus (DP) SCIT has been shown to be an effective and safe treatment for allergic rhinitis and allergic asthma [4]. Moreover, after end of 3 years of DP-SCIT treatment, the efficacy is sustained for at least an additional 2 years [5-7].

Allergen-specific IgG4 is functionally monovalent presumably preventing both IgE allergen interaction and allergen-IgE complex formation [8]. Due to its ability to compete with IgE for allergen binding, it is recognized as an IgE-blocking antibody and a marker of immune tolerance [9]. Previous studies showed that DP-SCIT induced a substantial increase in allergen-specific IgG4 during treatment [10]. Furthermore, it also increases IgE-blocking activities, which can be measured as IgE blocking factor (IgE-BF) [11]. The IgE-BF assay is a method that measures the competition between non-IgE and IgE antibodies for the binding to allergen. The majority of the IgE blocking activity can be explained by allergen-specific IgG4, but allergen-specific IgA antibodies may also contribute.

$\operatorname{Ig} \mathrm{A}$ is the quantitatively dominating immunoglobulin in the human body. It has 2 isotypes, IgA1 and IgA2, where IgA1 is predominantly present in serum, and IgA2 is exported to mucosal antibodies for the immune defense to inhaled and ingested antigens [12]. Thus, IgA is primarily present in serum in the form of $\operatorname{IgA} 1$ and in saliva in the form of IgA2. An important aspect of the mechanism underlying the clinical benefit of AIT is ascribed to the allergen blocking effect. It is therefore of interest to investigate whether IgA contributes to the blocking effect, and whether this potential blocking effect is correlated to that of allergen-specific IgG4.

The previously published studies on the change of allergen-specific IgG4 in AIT were mainly based on serological studies. Saliva is a special fluid that may be used as an alternative sampling method for investigating the change of IgG4 and IgA during AIT. The collection of saliva is a noninvasive method, easy to collect, and favorable for children. So far only 2 studies have investigated salivary DP-specific IgG4 and IgA relates to DP allergy. Miranda et al. [13] focused on the prevalence in allergic and nonallergic population and Liu et al. [14] reported on the treatment effect of SCIT comparing averages in groups without describing data from individual patients. However, no validated quantitative method was used for these studies; the result was reported in EI or AU/mL. Therefore, the aim of this study is to explore the allergen-specific IgE blocking antibodies induced by DP-SCIT treatment, in particular DP major allergen-specific IgG4 and IgA in serum and saliva and their correlation with DP IgE blocking activity.

\section{Material and Methods}

\section{Patients}

$286 \mathrm{HDM}$ allergic rhinitis subjects with/without asthma (5-50 years old) were recruited for DP-SCIT treatment in the ENT or pediatric clinic of 6 different hospitals in Zhejiang, Jiangsu, Shandong, Henan, and Hebei provinces. All patients fulfilled the ARIA guideline for allergic rhinitis and/or GINA guidelines for mild to moderate asthma. All patients were skin prick test-positive to HDM (DP or Dermatophagoides farinae, DF) (ALK-Abello A/S, Denmark) and had serum IgE against HDM (DP or DF) $>0.7 \mathrm{kU} / \mathrm{L}$. All subjects were given the necessary symptom-relieving medication during the study year. An open control group consisting of 21 HDM allergic children using symptom-relieving medication, but not receiving SCIT, was included in the study.

\section{SCIT Protocol}

Patients were treated with subcutaneous injections of standardized aluminum-formulated HDM DP Alutard ${ }^{\circledR}$ SQ vaccine (ALK-Abello A/S, Hørsholm, Denmark). The treatment protocol followed the recommended up-dosing schedule of 15 weeks before reaching the maintenance dose of 100.000 Alutard ${ }^{\circledR}$ SQ given every 6-8 weeks for 3 years. The data in this study were collected during the first year of SCIT treatment.

\section{Clinical Assessment}

Patients were asked to rate their symptoms and record the medication use over the past week at each research visit during the study period. The symptom and mediation use were scored according to WAO guideline [15]. Patients were asked to rate the symptoms of nose (blocking, itching, running nose, and sneezing), 
eyes (itching, hyperemia, swelling, and tearing), and chest (wheeze, cough, chest tightness, and shortness of breath) in the scale of $0-3$ (from $0=$ absent to 3 = severe). The mean score of nose, eyes, and chest symptoms was calculated individually. The sum scores of all 3 symptoms were the total symptom scores.

According to WAO recommendations [15], symptom-relieving medication use was scored as follows: 1 point was assigned to each dose of antihistamine, antileukotriene, or inhaled $\beta 2$-agonist; 2 points were assigned for each use of inhaled or nasal corticosteroids; and 3 points were assigned for the use of an oral corticosteroid. The daily medication score (MS) was the sum of each medication used in a day, and the mean of 7 days was used as the score for the week. The combined symptom and MS (CSMS) was calculated as the sum of symptom scores and MS.

The patients were asked to complete QoL questionnaires regarding rhinoconjunctivitis (RQLQ) and asthma (AQLQ) $[16,17]$ in the past week at each research visit. There are 28 questions in RQLQ, and 31 questions in AQLQ. Scores were assigned in a range of $0-6$ for both RQLQ and AQLQ $(0=$ best possible and $6=$ worse possible). The mean score of all questions in each questionnaire was recorded. The sum of RQLQ and AQLQ is referred to as the total QoLS.

\section{Sample Collection Timepoint}

Saliva and serum samples were collected from each patient in the SCIT group at 3 timepoints: before treatment, at the end of the SCIT up-dosing phase ( 4 months SCIT), and after an additional 8 months of maintenance treatment (12 months SCIT in total). Saliva and serum samples were collected from each patient in the control group at 2 timepoints: before treatment and after 12 months of treatment. Samples were stored at $-20^{\circ} \mathrm{C}$ before measurement.

\section{Saliva Collection}

Saliva samples were collected during clinic visits and under the instruction of clinical professionals. The saliva collection device used was Salivette ${ }^{\circledR}$ (SARSTED AG \& Co., Numbrecht, Germany), according to the manufacturer's instructions. The swab from Salivette ${ }^{\circledR}$ was placed in the mouth for $5 \mathrm{~min}$ and then reinserted in the Salivette ${ }^{\circledR}$. The Salivettes were stored at $-20^{\circ} \mathrm{C}$ before centrifugation. Centrifuged at 3,000 $\mathrm{g}$ for $10 \mathrm{~min}$. Clarified saliva samples were aliquoted and stored at $-20^{\circ} \mathrm{C}$ before use.

\section{Allergen-Specific IgE and IgE-BF}

DP-, DF-, Der p 1-, and Der p 2-specific IgE were measured using the ADVIA Centaur ${ }^{\circledR}$ immunoassay system (Siemens AG, Erlangen, Germany), a reverse sandwich immunoassay using direct chemiluminescent technology [18]. Results were reported as $\mathrm{kUA} / \mathrm{L}$. Specific IgE results were calibrated against ImmunoCAPspecific IgE. The lower limit of quantification (LoQ) of specific IgEs was $0.35 \mathrm{kUA} / \mathrm{L}$. A result $>0.35 \mathrm{kUA} / \mathrm{L}$ was considered positive.

DP- and DF-specific IgE-BF were measured using the ADVIA Centaur ${ }^{\circledR}$ immunoassay system (Siemens AG, Erlangen, Germany), using a previously reported method [11]. Briefly, the IgE-BF method used 2 different assay procedures: one for normal IgE determination which includes a wash step after serum incubation for removing all serum components except the bound IgE before adding the biotinylated antigen; the other is "competition" IgE determination, which excludes the wash step after serum incuba- tion, and allows the non-IgE antibodies to compete with IgE in binding to biotinylated antigen. The calculation is $\operatorname{IgE}-\mathrm{BF}=1-$ $\left(\mathrm{IgE}_{\text {competition }} / \operatorname{IgE} \mathrm{E}_{\text {normal }}\right)$. IgE-BF values $<0.1$ were defined as no blocking activity because the variation of IgE measurement in $\mathrm{AD}$ VIA Centaur ${ }^{\circledR}$ system is $10 \%$.

\section{Allergen-Specific IgG4}

DP-, Der p 1-, and Der p 2-specific IgG4 in serum or saliva samples were measured by sandwich ELISA as reported previously [10]. In brief, the microplate was coated with $10 \mu \mathrm{g} / \mathrm{mL}$ DP extract (ALK-Abello, Denmark), $2 \mu \mathrm{g} / \mathrm{mL}$ nDer $\mathrm{p} 1$ (purified inhouse by an affinity column pre-coated with anti-Der $\mathrm{p} 1$ rabbit antibody) or $2 \mu \mathrm{g} / \mathrm{mL}$ rDer p 2 [19] protein (rDer p 2.0101 isoallergen). Then blocked with $2 \%$ casein, and subsequently incubated with diluted standards, controls, and serum samples (in $0.5 \%$ casein). Mouse mAbs to human IgG4 (ALK-Abello, Denmark) was used at 1/10,000 for detection, and revealed by HRP labeled goat anti-mouse IgG $(1 / 20,000$; KPL, USA), then followed by Tetramethylbenzidine visualizing. Assay conditions were optimized to be suitable for measuring saliva samples.

The assays were further calibrated against ImmunoCAP-specific IgG4 (Uppsala, Sweden) by measuring the specific IgG4 concentration in mgA/L of DP, Der p 1, and Der p 2 IgG4 in the ELISA standard stock solutions by ImmunoCAP, and the results were reported in $\mathrm{mg} / \mathrm{L}$. The lower LoQ of serum DP, Der p 1, and Der p 2 IgG4 was 3.33, 1.52, and $1.92 \mu \mathrm{g} / \mathrm{L}$, respectively. The LoQ of salivary DP, Der p 1, and Der p 2 IgG4 was $0.67,0.30$, and 0.38 $\mu \mathrm{g} / \mathrm{L}$, respectively. The difference in LoQ of serum and saliva was due to the different matrix effects in serum and saliva. Serum samples needed to be diluted at least 5-fold in order to remove the matrix effect, whereas there was no need to dilute saliva samples.

\section{Allergen-Specific IgA1 and IgA2}

Der p 2-specific IgA1 and IgA2 in serum and saliva were measured by four-layer double antibodies sandwich ELISA. The microplate was coated with $10 \mu \mathrm{g} / \mathrm{mL}$ of the Der p 2 protein (ALKAbello, Denmark). Blocked with 0.5\% Lysozyme (Sigma-Aldrich, USA) and then incubated with 40 -fold diluted serum or 5 -fold diluted saliva (in $0.125 \%$ Lysozyme) samples. Mouse mAb to IgA1 (clone C3507B5; Creative Diagnostics, USA) or IgA2 (clone B9605D3; Creative Diagnostics, USA) were used at $0.5 \mu \mathrm{g} / \mathrm{mL}$ for detection and revealed by HRP labeled goat anti-mouse IgG (KPL, USA) followed by Tetramethylbenzidine for visualizing.

The assay used recombinant standard IgA1 or IgA2 anti-Der $\mathrm{p}$ 2 which were made as follows: the variable Der $\mathrm{p} 2$ binding domains were cloned from a human subject via phage display as previously described [19] and converted into human IgA1 and IgA2 by insertion of IGHA1 (P01876) and IGHA2 (P01877), respectively. Antibodies were purified via size exclusion chromatography, and the titers were determined against an IgA standard using a GyroLab assay (Uppsala, Sweden).

The assay standard was diluted in serum or saliva pool to ensure a common sample matrix. The results are reported in $\mu \mathrm{g} / \mathrm{L}$. The lower LoQ of Der p 2-specific IgA1 and IgA2 in serum are both $5.6 \mu \mathrm{g} / \mathrm{L}$, while the LoQ in saliva is both $0.7 \mu \mathrm{g} / \mathrm{L}$. The difference of LoQ in serum and saliva is due to the different matrix effects in serum and saliva. Serum samples need to be diluted 40 -fold, and saliva samples need to be diluted 5-fold, in order to reduce nonspecific binding from the matrix. 
Table 1. Demographic data and clinical characteristics before treatment

\begin{tabular}{|c|c|c|c|}
\hline & SCIT group & Control group & $p$ value $^{1}$ \\
\hline$N$ & 286 & 21 & - \\
\hline Age, yr, median $\left(\right.$ range $\left.^{2}\right)$ & $9(5-48)$ & $10(5-50)$ & 0.306 \\
\hline Gender (male/female, $n / n$ ) & $197 / 89$ & $8 / 13$ & - \\
\hline Diagnosis $(\mathrm{AR} / \mathrm{AR}+\mathrm{AS}, n / n)$ & $139 / 147$ & $9 / 12$ & - \\
\hline CSMS, mean (95\% CL of mean) & $4.1(3.9-4.3)$ & $3.1(2.0-4.1)$ & 0.131 \\
\hline QoL scores, mean (95\% CL of mean) & $2.2(2.0-2.4)$ & $1.6(0.9-2.3)$ & 0.163 \\
\hline \multicolumn{4}{|l|}{ IgE, kU/L, median (interquartile range ${ }^{3}$ ) } \\
\hline DP IgE & $82.6(28-183)$ & $34.0(6-71)$ & 0.056 \\
\hline DF IgE & $82.6(28-183)$ & $34.0(6-71)$ & 0.056 \\
\hline Der p $1 \operatorname{IgE}$ & $37.8(8-67)$ & $6.0(0.8-36)$ & 0.066 \\
\hline Der p 2 IgE & $36.6(10-73)$ & $21.3(6-55)$ & 0.670 \\
\hline \multicolumn{4}{|c|}{ Serum IgG4, $\mu \mathrm{g} / \mathrm{L}$, median (interquartile range) } \\
\hline DP IgG4 & $148(71-306)$ & $120(46-148)$ & 0.047 \\
\hline Der p 1 IgG4 & $31(7.2-71)$ & $13(2.7-32)$ & 0.060 \\
\hline Der p 2 IgG4 & $40(12-99)$ & $13(5.5-43)$ & 0.047 \\
\hline \multicolumn{4}{|c|}{ Salivary IgG4, $\mu \mathrm{g} / \mathrm{L}$, median (range), Detectable, $\%$} \\
\hline $\mathrm{DP}$ & $0.3(0.3-14), 11$ & $0.3(0.3-0.3), 0$ & 0.142 \\
\hline Der p 1 & $0.2(0.2-6), 10$ & $0.2(0.2-0.2), 0$ & 0.170 \\
\hline Der p 2 & $0.2(0.2-5), 3.4$ & $0.2(0.2-0.2), 0$ & 0.416 \\
\hline \multicolumn{4}{|c|}{ Serum IgA, $\mu g / L$, median (interquartile range) } \\
\hline Der p 2 IgA1 & $28(10-46)$ & $17(2.8-35)$ & 0.278 \\
\hline Der p 2 IgA2 & $2.8(2.8-2.8)$ & $2.8(2.8-2.8)$ & 0.465 \\
\hline \multicolumn{4}{|c|}{ Salivary IgA, $\mu \mathrm{g} / \mathrm{L}$, median (interquartile range) } \\
\hline Der p $2 \operatorname{IgA} 1$ & $0.4(0.4-3.4)$ & $0.4(0.4-0.9)$ & 0.750 \\
\hline Der p 2 IgA2 & $0.4(0.4-0.9)$ & $0.4(0.4-0.8)$ & 0.632 \\
\hline \multicolumn{4}{|l|}{ IgE-BF, median (interquartile range) } \\
\hline DP IgE-BF & $-0.08(-0.18$ to 0.01$)$ & $-0.02(-0.27$ to 0.10$)$ & 0.782 \\
\hline DF IgE-BF & $-0.14(-0.24$ to 0$)$ & $-0.06(-0.27$ to 0.01$)$ & 0.858 \\
\hline
\end{tabular}

SCIT, subcutaneous immunotherapy; DP, Dermatophagoides pteronyssinus; DF, Dermatophagoides farinae; CSMS, combined symptom and medication scores; CL, confidence limit. ${ }^{1}$ Comparisons between the 2 treatment groups using the Tukey-Kramer HSD method. Data that were not normally distributed underwent log transformation before the comparison. $p<0.05$ was considered statistically significant. ${ }^{2}$ Range was represented by the range of minimum to maximum. ${ }^{3}$ Interquartile range was represented by $25-75 \%$ quartile.

\section{Antibody Depletion in Patient Sera after DP SCIT}

Antibody depletion experiments were performed on 12 sera obtained after 12 months DP SCIT treatment with IgE-BF levels in the range of $0.3-0.5$ to identify the antibody isotype primarily responsible for IgE blocking activity. The antibody depletion was performed using the Magrose Protein G (Pro-G) Immunoprecipitation beads (BEAVER Biomedical, China) according to the manufacturer's instruction. An IgG depletion procedure with 2 rounds of Pro-G depletion was performed in order to achieve a substantial reduction of DP IgE-BF. The procedure in detail: take out $0.25 \mathrm{~mL}$ serum and incubate with $0.25 \mathrm{~mL}$ Pro-G beads for $15 \mathrm{~min}$ for the first round of depletion; then transfer the serum to another $0.25 \mathrm{~mL}$ new Pro-G beads and incubated $15 \mathrm{~min}$ for second round of depletion. Collect the serum after depletion, and then it is ready for analyses. The same serum was treated with dummy Magrose beads (the binding site of beads was blocked by the blocking buffer before adding to serum) to establish the level of "non-specific binding" during depletion. DP IgE-BF; DP-, Der p 1-, and Der p 2-specific IgG4 and Der p 2-specific IgA levels before and after depletion were measured. IgA data are repre- sented as the sum of Der p 2-specific IgA1 and IgA2, in order to determine the effect of depletion on Der $\mathrm{p}$ 2-specific IgA levels. The depletion results of DP IgE-BF are presented for 3 conditions (initial, dummy depletion, and Protein G depletion), whereas IgG4 and IgA are presented as the ratio relative to the initial level.

\section{Statistical Analysis}

Samples with IgE, IgG4, IgA1, and IgA2 results below LoQ were reported as half of the LoQ. The change from baseline of CSMS, QoL, serum IgE, IgG4, IgA, and salivary IgG4, and IgA were calculated as a log-transformed ratio, while the change of baseline of IgE-BF was calculated as a delta value.

The change from baseline of clinical responses (CSMS and QoL) and immunological responses (specific IgE, IgE-BF, and IgG4) over treatment time were fitted to a repeated measures linear mixed model assuming compound symmetry. The models were set up and analyzed with JMP add-in "Full factorial repeated measures ANOVA add-In." The least square means data were imported to GraphPad Prism and were transformed back, then plotted on original 


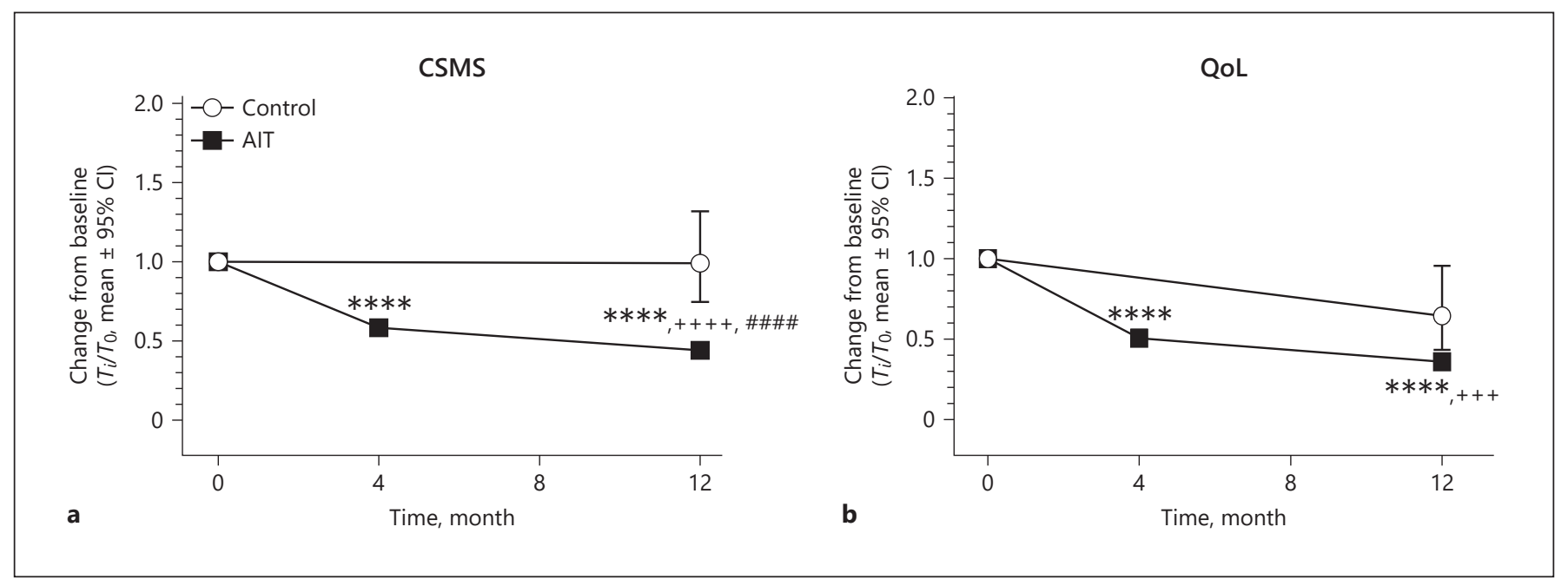

Fig. 1. The change from baseline in clinical response during treatment. CSMS (a); total QoL scores (b). The change from baseline in clinical scores was calculated as the ratio between after treatment $\left(T_{\mathrm{i}}\right)$ and before treatment $\left(T_{0}\right)$. As ratios were not normal distributed, they have been log 10 transformed before fitting into linear mixed models. After the model fitting, the least square mean and $\mathrm{CL}$ were obtained before they were transformed $\left(10^{\wedge}\right)$ back to the original scale. Turkey's HSD test was used for multiple com- parisons. The differences between timepoints $\left(T_{\mathrm{i}}\right)$ compared to baseline $\left(T_{0}\right)$ are shown as $p<0.0001$ : “****." The difference between timepoint 4 and 12 months is shown as $p<0.001$ : “+++," $p<0.0001:$ " ++++ ." The difference between SCIT and control is shown as $p<0.0001$ : “\#\#\#\#." SCIT, subcutaneous immunotherapy; CL, confidence limit; CSMS, combined symptom and medication scores; QoL, quality of life.

1 or Der p 2, while $84 \%$ were sensitized to both. In the control group, all patients were sensitized to either Der $\mathrm{p}$ 1 or Der $\mathrm{p} 2$ and $71 \%(15 / 21)$ were sensitized to both. The level of IgE sensitization was comparable in SCIT and control patients. The SCIT group had a slightly higher serum-specific IgG4 level against DP and Der p 2, whereas there was no difference in Der p 1-specific IgG4 level. No difference between the SCIT and control group was found in specific IgA or IgE-BF.

\section{Clinical Benefit}

Clinical responses were evaluated based on an individual's change compared to baseline. The CSMS in the SCIT group was significantly reduced at both 4 and 12 months of SCIT, while there was no change in the control group (Fig. 1a). After 12 months of treatment, the least square mean ratio $\left(T_{\mathrm{i}=12} / T_{\mathrm{i}=0}\right)$ of CSMS in SCIT group was 0.44 , indicating an average improvement in the SCIT group of $56 \%$. The active group was statistically different from the control group which further showed no development in CSMS over the 12 months of treatment.

The QoL scores in the SCIT group were significantly decreased at both 4 and 12 months of SCIT, whereas there was no change in the control group (Fig. 1b). At 12 months of treatment, the mean ratio QoL scores of the 


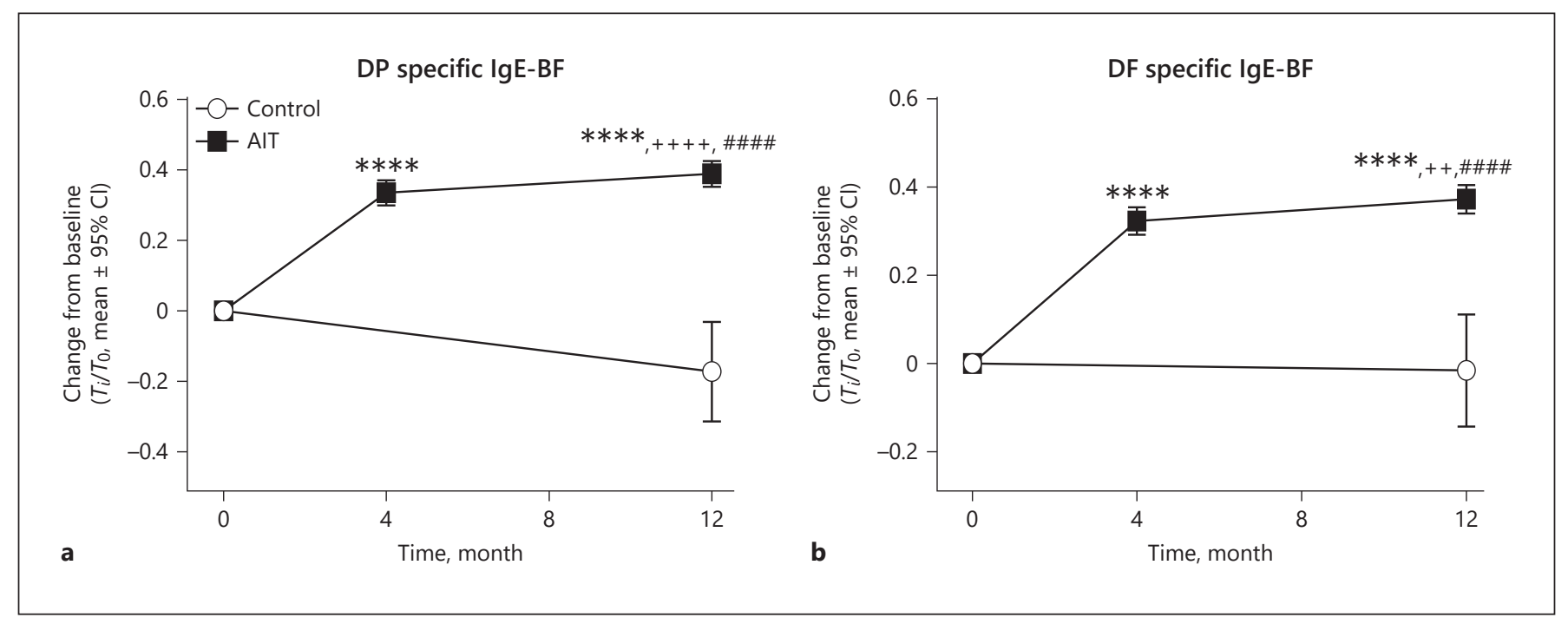

Fig. 2. The change from baseline in allergen-specific IgE-BF (BF) during SCIT. The change from baseline of IgE-BF was calculated as the difference in $I g E-B F_{\text {after treatment }}-\operatorname{IgE}-\mathrm{BF}_{\text {befrore treatment. }} \mathrm{DP}$ specific IgE-BF (a); DF-specific IgE-BF (b). Turkey's HSD test was used for multiple comparisons in IgE-BF. The differences between timepoints $\left(T_{\mathrm{i}}\right)$ compared to baseline $\left(T_{0}\right)$ are shown as $p<$

SCIT group was 0.36 , so the improvement was $64 \%$. Whereas the mean ratio of control group was 0.65 , corresponding to $35 \%$ improvement. Due to small sample size and large variation in the control group, no significant difference was found between 12 months and baseline or difference between control and SCIT group at 12 months. Therefore, the control group would mainly serve as immunological reference in this study.

\section{Immunological Responses in Serum}

In the SCIT group allergen-specific IgE exhibit a small increase of DP- and Der p 1-, Der p 2-specific $\operatorname{IgE}(23,38$, and $17 \%$, respectively) after 4 months of treatment, but no further increase after 12 months. There was no change in any of the tested specific IgEs in the control group (data not shown).

For the blocking of IgE binding to allergen, DP-specific IgE-BF increased significantly at 4 and 12 months in the SCIT treatment group. The largest changes were observed after 4 months of SCIT. There were no changes in the control group (Fig. 2a). Although the SCIT treatment only used DP extract, the DF-specific IgE-BF exhibits an identical trend as DP-specific IgE-BF (Fig. 2b). There is a strong correlation between DF IgE-BF and DP IgE-BF at all SCIT treatment timepoints (Fig. 3a), and DF IgE is also
0.0001:“****." The difference between timepoint 4 and 12 months are shown as $p<0.001$ : “+++," $p<0.0001:$ : ++++ ." The difference between SCIT and control is shown as $p<0.0001$ :"\#\#\#\#." SCIT, subcutaneous immunotherapy; DP, Dermatophagoides pteronyssinus; DF, Dermatophagoides farinae; IgE-BF, IgE blocking factor.

strongly correlated with DP IgE (Fig. 3b), possibly due to extensive cross-reactivity.

Before treatment initiation, serum DP-specific IgG4 was detected in all subjects, Der p 1-, and Der p 2-specific IgG4 were detected in $90 \%$ subjects in the SCIT group. After SCIT, all patients had detectable specific IgG4. DP-, Der p 1-, and Der p 2-specific IgG4 were significantly increased: approximately 9,12 , and 7 -fold in the SCIT group at 4 months, and a further increase to 29 , 57 , and 20 -fold at 12 months of SCIT. No change in IgG4 levels was detected in the control group. The specific IgG4 after treatment in the SCIT group was significantly higher than in the control group, see Fig. $4 \mathrm{a}-\mathrm{c}$.

Serum Der p 2-specific IgA1 antibodies were detected in 82 and $71 \%$ of the patients in the SCIT and control groups, respectively before treatment. There were no changes in Der p 2-specific IgA1 in serum during the treatment, and similar levels were observed in both SCIT and control group, shown in Figure 4d.

Comparing the Der p 2-specific IgG4 and IgA1 in serum, IgG4 and IgA1 were in the same range before treatment, and the median level was 40 and $28 \mu \mathrm{g} / \mathrm{mL}$, respectively (Table 1). After treatment, the IgG4 level was 10 and 25 times higher than IgA 1 at 4 and 12 months of DPSCIT, respectively, shown in Fig. $4 c-d$. 
Fig. 3. The correlation between DF- and $\mathrm{DP}$-specific IgE-BF and IgE. Correlation of DF IgE-BF versus DP IgE-BF (a); correlation of Log10 DF IgE versus Log10 DP IgE (b). The correlation coefficient and its $p$ value were calculated by Pearson correlations. DP, Dermatophagoides pteronyssinus; DF, Dermatophagoides farinae; IgE$\mathrm{BF}$, IgE blocking factor.

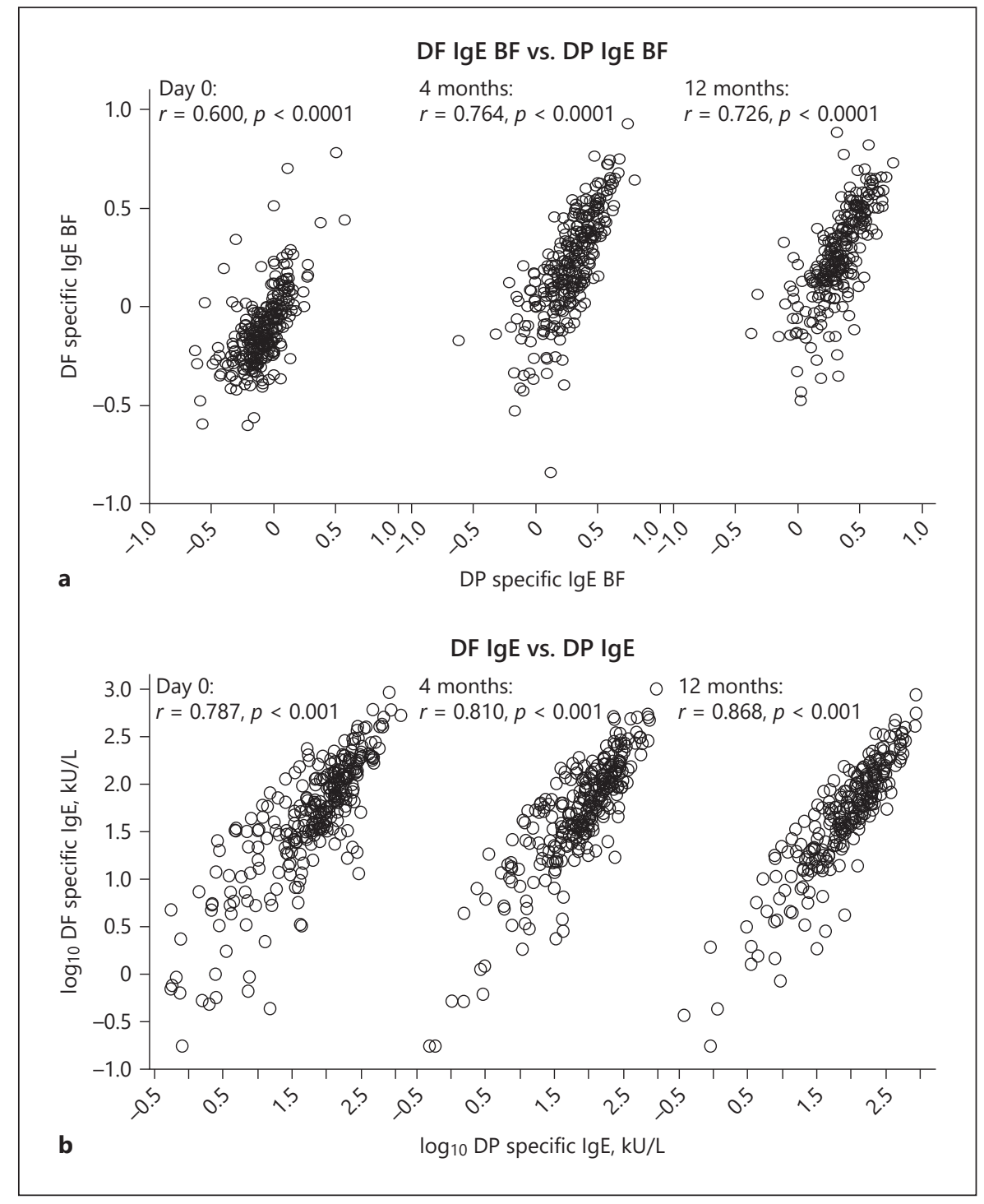

Der p 2-specific IgA2 was detectable in 18\% (49/277) of serum samples. There was a small but significant change in serum Der $\mathrm{p} 2$-specific IgA2 antibodies at 4 and 12 months of SCIT treatment, but no change in control group, see Figure 4e. The median increase in the ratio of serum Der p 2-specific IgA2 was 2.4 and 2.7 at 4 and 12 months of DP-SCIT, respectively. No difference was observed in the IgA2 level between SCIT and control group at 12 months of treatment.

\section{Immunological Responses in Saliva}

After 12 months of SCIT, 76\% (180/238) had measurable DP IgG4, 66\% (156/238) had measurable Der p 1 IgG4, and 37\% (87/238) had measurable Der p 2 IgG4. Even though before SCIT, the measurable DP-, Der p 1-, and Der p 2-specific IgG4 was only 11,10 , and 3.5\%, respectively, and at 4 months of SCIT, they become 42,30 , and $17 \%$, respectively. Salivary DP-, Der p 1-, and Der p 2 -specific IgG4 showed significant increases at both 4 and 12 months of SCIT. The average increase in DP-, Der $\mathrm{p}$ 1-, and Der p 2-specific IgG4 at 4 months was 1.8, 1.5, and 1.2 -fold, respectively. At 12 months, the increase ratio was 5.4, 4.1, and 1.8-fold, respectively. No change was detected in the control group (Fig. 5a-c). The salivary-specific IgG4 levels in the SCIT group were significantly higher than in the control group at 12 months.

Salivary Der p 2-specific IgA 1 could be detected in $38 \%$ $(106 / 277)$ of patients in the SCIT group and 24\% (5/21) of patients in the control group before treatment. After treatment, no change was detected in neither the SCIT 


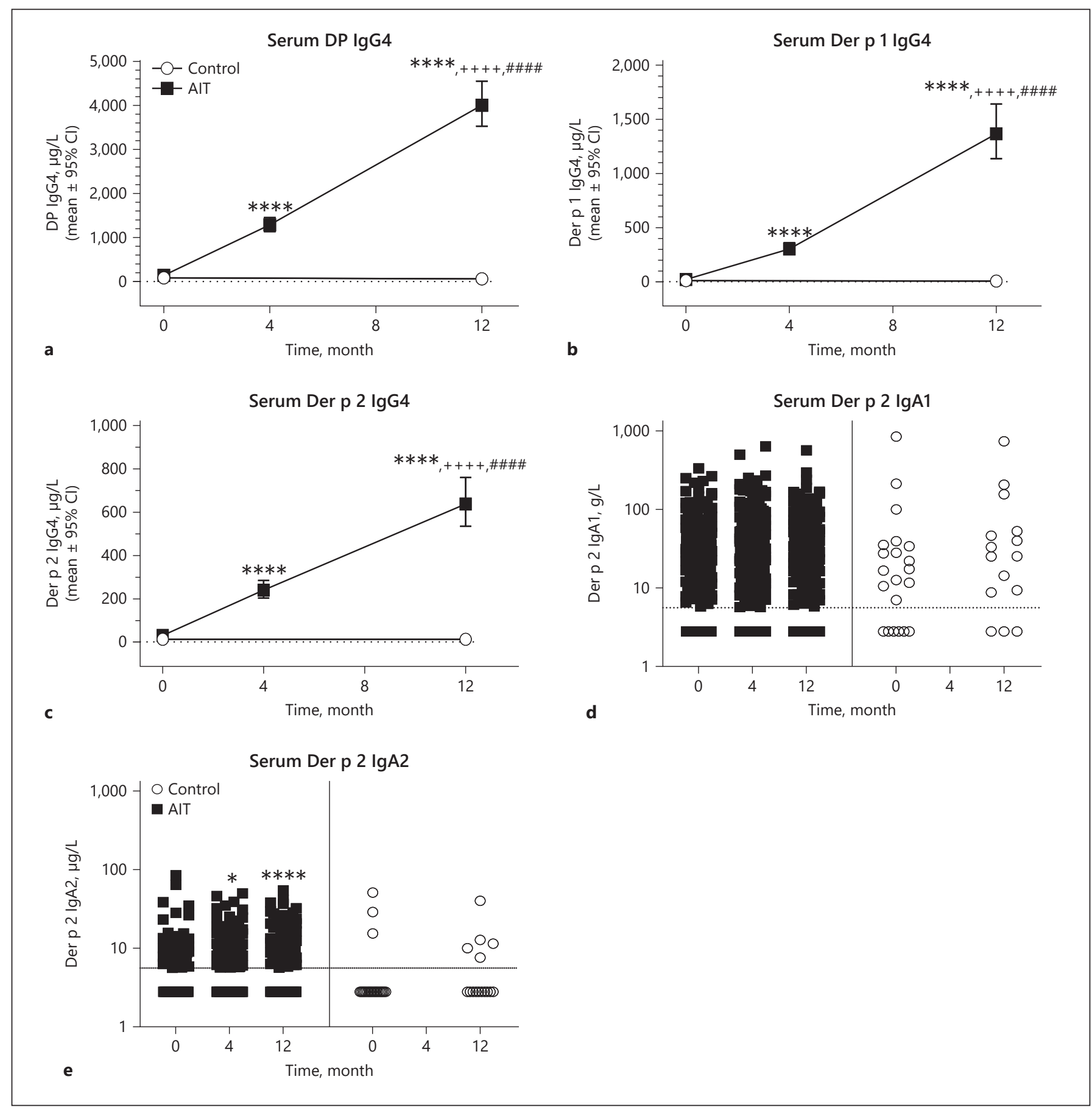

Fig. 4. The change of allergen-specific IgG4 and IgA in serum during the treatment. DP-specific IgG4 (a); Der p 1-specific IgG4 (b); Der p 2-specific IgG4 (c); Der p 2-specific IgA1 (d); Der p 2-specific IgA2 (e). The horizontal dashed line is the LOQ of each assay. Turkey's HSD test was used for multiple comparisons in (a-c). The differences between timepoints $\left(T_{\mathrm{i}}\right)$ compared to baseline $\left(T_{0}\right)$ are

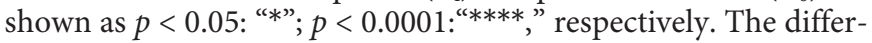

ence between timepoint 4 and 12 months is shown as $p<0.01$ : “++," $p<0.0001:$ : ++++." The difference between SCIT and control is shown as $p<0.0001$ : “\#\#\#\#." The Friedman test was used for comparison in (d, e). No difference was found between different timepoints. SCIT, subcutaneous immunotherapy; DP, Dermatophagoides pteronyssinus. 


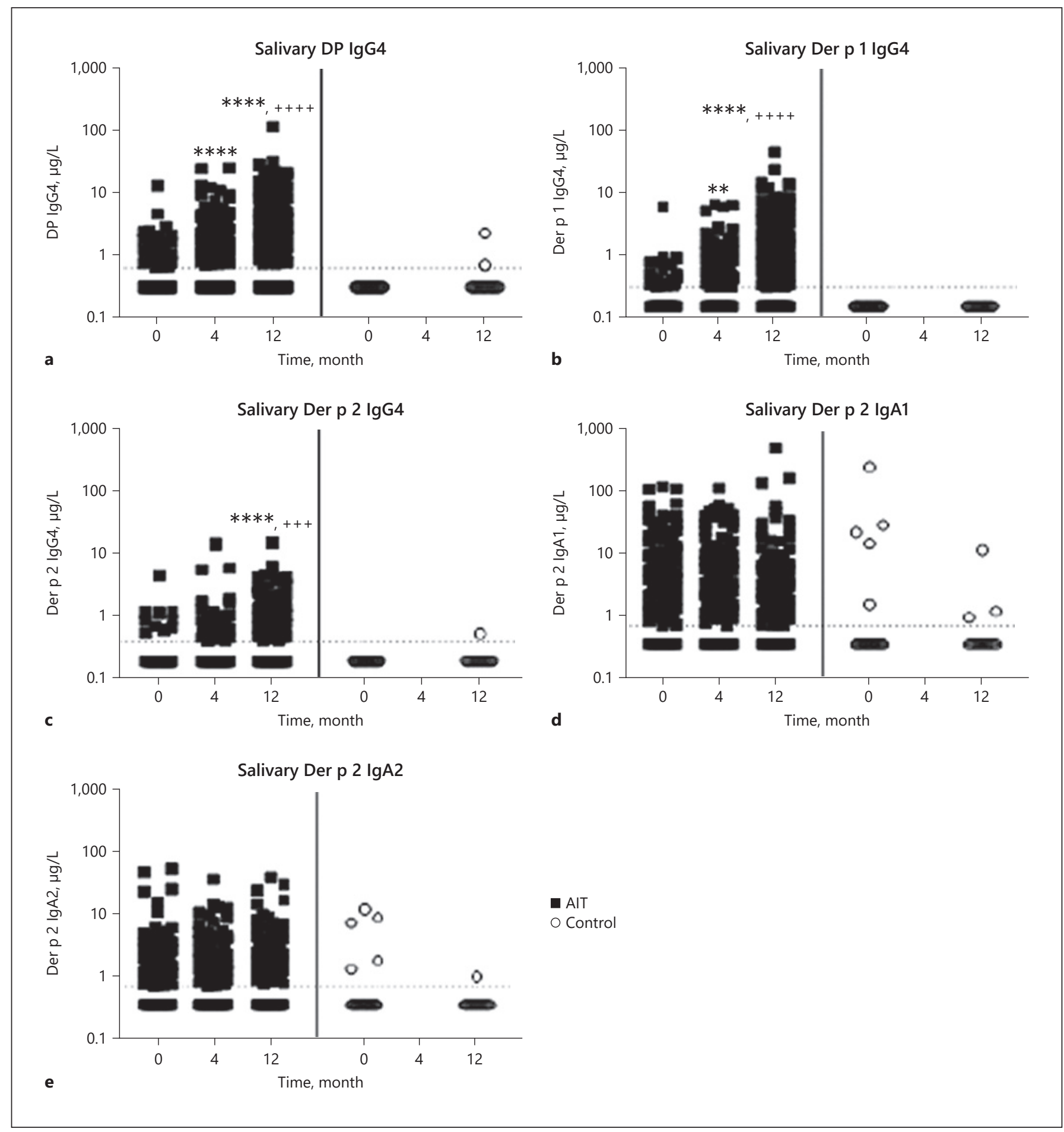

Fig. 5. The change of allergen-specific IgG4 and IgA in saliva during treatment. DP-specific IgG4 (a); Der p 1-specific IgG4 (b); Der p 2-specific IgG4 (c); Der p 2-specific IgA1 (d); Der p 2-specific IgA2 (e). The horizontal dashed line is the LOQ of each assay. The Friedman test was used for comparing the difference between timepoints in the SCIT group. The differences between timepoints
$\left(T_{\mathrm{i}}\right)$ compared to baseline $\left(T_{0}\right)$ are shown as $p<0.01$ : “**”; $p<$ 0.0001 : “****," respectively. The difference between timepoint 4 and 12 months is shown as $p<0.001$ : “+++," $p<0.0001$ :“++++." SCIT, subcutaneous immunotherapy; DP, Dermatophagoides pteronyssinus. 
Table 2. Correlation of IgG4 and IgA between saliva and serum at different SCIT treatment timepoints

\begin{tabular}{|c|c|c|c|c|c|c|c|c|c|}
\hline \multirow{2}{*}{$\begin{array}{l}\text { Correlation }{ }^{1} \text { between } \\
\text { saliva and serum }\end{array}$} & \multicolumn{3}{|l|}{$0 \mathrm{mo}$} & \multicolumn{3}{|l|}{$4 \mathrm{mo}$} & \multicolumn{3}{|l|}{$12 \mathrm{mo}$} \\
\hline & $n(\%)$ & $r$ & $p$ value & $n(\%)$ & $r$ & $p$ value & $n(\%)$ & $r$ & $p$ value \\
\hline DP IgG4 & $32(11)$ & 0.079 & 0.667 & $117(42)$ & 0.271 & $0.0032^{*}$ & $201(82)$ & 0.437 & $<0.0001^{*}$ \\
\hline Der p 1 IgG4 & $28(11)$ & 0.367 & 0.051 & $88(31)$ & 0.322 & $0.0023^{*}$ & $170(71)$ & 0.503 & $<0.0001^{*}$ \\
\hline Der p 2 IgG4 & $10(4)$ & -0.117 & 0.747 & $46(17)$ & 0.257 & 0.0851 & $93(39)$ & 0.407 & $<0.0001^{*}$ \\
\hline Der p 2 IgA 1 & $85(36)$ & 0.151 & 0.168 & $90(38)$ & 0.159 & 0.135 & $94(44)$ & 0.129 & 0.214 \\
\hline Der p 2 IgA2 & $13(27)$ & 0.299 & 0.321 & $27(30)$ & 0.102 & 0.612 & $34(36)$ & 0.065 & 0.716 \\
\hline
\end{tabular}

Significant correlation is highlighted with “*” in $p$ value. SCIT, subcutaneous immunotherapy; DP, Dermatophagoides pteronyssinus. ${ }^{1}$ The Pearson correlation is only calculated on the samples $>$ LOQ in both saliva and serum. " $n(\%)$ " is the number of samples that were used for the calculation, and the percentage of total number of samples that larger than LOQ in serum. The IgG4 and IgA data were log 10 transformed before calculating the correlation.

nor the control group. No differences between groups were detected at 12 months, see Figure $5 \mathrm{~d}$.

Salivary Der p 2-specific IgA2 could be detected in $26 \%$ $(72 / 277)$ of the SCIT patients and $24 \%(5 / 21)$ of the control patients before treatment. Similar to salivary IgA1, no change was detected after treatment in neither the SCIT nor control group, and no difference between the SCIT and control group was detected at 12 months of treatment.

Comparing the salivary Der p 2-specific IgG4 and $\operatorname{IgA} 1 / \operatorname{Ig} A 2$ in the SCIT group, IgA1 and IgA2 seemed to be the dominating Der p 2-specific antibody in saliva, since it was 5.2 and 2.8 times of the amount of IgG4 before treatment and 3.3 and 1.8 times the amount of IgG4 after 12 months of SCIT, see Figure 4a-d. However, there was no change in Der p 2-specific IgA1 and IgA2 after SCIT, whereas Der p 2-specific IgG4 increased significantly.

Correlation between Allergen-Specific IgG4 and IgA in Saliva and Serum

Table 2 shows the correlation of IgG4 and IgA between saliva and serum; only the measurable samples (>LoQ) in both saliva and serum were included. There was no correlation before treatment, but significant correlation of DP- and Der p 1-specific IgG4 were detected at 4 months of SCIT, and it became highly significant $(r=0.4-0.5)$ for DP, Der p 1, and Der p 2 at 12 months of SCIT. The correlation became stronger with longer SCIT duration. The correlation related to the number of measurable salivary IgG4 samples, the more measurable salivary samples, and the stronger correlation. The ratio of serum/saliva IgG4 at 12 months of DP-SCIT was about $1,300-1,500$. No samples of measurable IgG4 in saliva, but not in serum, were observed.

There was no correlation between salivary and serum IgA1 and IgA2 at any treatment timepoints. There were 10 times more positive samples in serum Der $\mathrm{p} 2$-specific IgA1 than in saliva (48 vs. $4.4 \%$ ). Der p 2 -specific IgA1 was dominant in serum compare to saliva. Whereas for Der p 2-specific IgA2, the number of measurable samples in serum and saliva were similar.

\section{Correlation of DP-Specific IgE-BF with Allergen-}

Specific IgG4 and IgA

There was a significant correlation $(r=0.31-0.62)$ between DP-specific IgE-BF and DP-, Der p 1-, and Der $p$ 2 -specific IgG4 in serum at all treatment timepoints, see Table 3. The correlation coefficient $(r)$ became larger with longer treatment time (all $r>0.5$ at 12 months). Salivary DP-, Der p 1-, and Der p 2-specific IgG4 showed a weak but significant correlation $(r=0.23-0.38)$ with DP IgE-BF at SCIT 12 months, which was associated with the number of positive samples. No significant Pearson correlation was obtained between DP-specific IgE-BF and Der $p$ 2 -specific IgA1 and IgA2 in neither serum nor saliva even though the Der p 2 IgA1 was measurable in most of the serum samples.

\section{The Effect of IgG4 Depletion on IgE-BF}

The results of depletion experiments are shown in Figure 6. It shows that DP IgE-BF remains unchanged after dummy bead depletion (dummy), while after Pro-G depletion, the IgE blocking effect was completely removed in 8 out of 12 sera (IgE-BF $<0.1$ ), see Figure 6 a. For IgG4, about $20 \%$ of specific IgG4 against DP, Der p 1, and Der 
Table 3. Correlation between allergen-specific IgE blocking antibodies concentration and DP IgE-BF at different SCIT treatment timepoints

\begin{tabular}{|c|c|c|c|c|c|c|c|c|c|}
\hline \multirow{2}{*}{$\begin{array}{l}\text { Correlation }{ }^{1} \text { of DP } \\
\text { IgE-BF }\end{array}$} & \multicolumn{3}{|c|}{$0 \mathrm{mo}$} & \multicolumn{3}{|c|}{$4 \mathrm{mo}$} & \multicolumn{3}{|c|}{$12 \mathrm{mo}$} \\
\hline & $n$ & $r$ & $p$ value & $n$ & $r$ & $p$ value & $n$ & $r$ & $p$ value \\
\hline \multicolumn{10}{|l|}{ Serum IgG4 } \\
\hline $\mathrm{DP}$ & 282 & 0.309 & $<0.0001^{*}$ & 280 & 0.391 & $<0.0001^{*}$ & 244 & 0.617 & $<0.0001^{*}$ \\
\hline Der p 1 & 254 & 0.375 & $<0.0001^{*}$ & 280 & 0.329 & $<0.0001^{*}$ & 240 & 0.526 & $<0.0001^{*}$ \\
\hline Der $\mathrm{p} 2$ & 258 & 0.317 & $<0.0001^{*}$ & 273 & 0.319 & $<0.0001^{*}$ & 241 & 0.562 & $<0.0001^{*}$ \\
\hline \multicolumn{10}{|l|}{ Salivary IgG4 } \\
\hline DP & 32 & 0.123 & 0.501 & 117 & 0.007 & 0.941 & 193 & 0.231 & $0.0012^{*}$ \\
\hline Der p 1 & 29 & 0.377 & $0.044^{*}$ & 88 & 0.069 & 0.522 & 165 & 0.271 & $0.0004^{*}$ \\
\hline Der $\mathrm{p} 2$ & 10 & 0.349 & 0.323 & 46 & 0.032 & 0.834 & 91 & 0.375 & $0.0002^{*}$ \\
\hline \multicolumn{10}{|l|}{ Serum IgA } \\
\hline Der p $2 \operatorname{IgA} 1$ & 234 & 0.069 & 0.299 & 239 & 0.013 & 0.846 & 216 & 0.039 & 0.575 \\
\hline Der p 2 IgA2 & 49 & -0.176 & 0.236 & 90 & 0.099 & 0.354 & 95 & 0.091 & 0.382 \\
\hline \multicolumn{10}{|l|}{ Salivary IgA } \\
\hline Der p $2 \operatorname{IgA} 1$ & 106 & -0.116 & 0.267 & 111 & 0.070 & 0.482 & 106 & -0.082 & 0.407 \\
\hline Der p 2 IgA2 & 72 & 0.114 & 0.332 & 72 & -0.098 & 0.397 & 80 & -0.042 & 0.711 \\
\hline
\end{tabular}

Significant correlation is highlighted with “*” in $p$ value. SCIT, subcutaneous immunotherapy; DP, Dermatophagoides pteronyssinus; IgE-BF, IgE blocking factor. ${ }^{1}$ The Pearson correlation is only calculated on the samples $>$ LOQ in both saliva and serum. The " $n$ " is the number of samples that were used for the calculation. The IgG4 and IgA data were log 10 transformed before calculating the correlation.

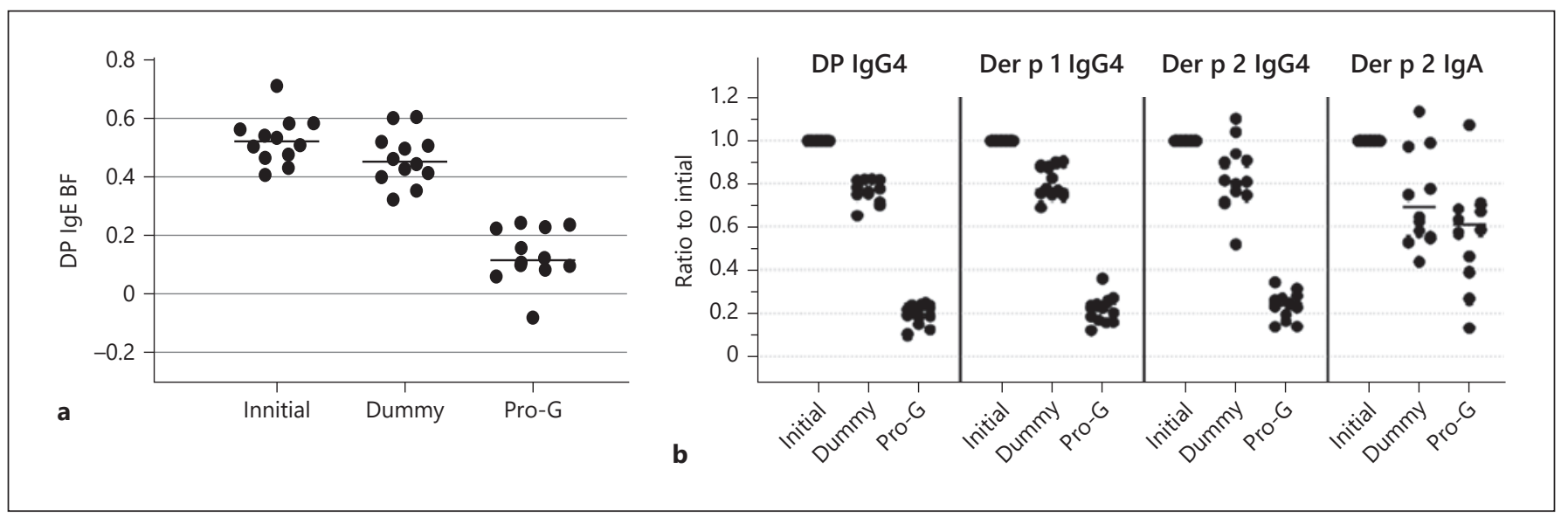

Fig. 6. Depletion effect on DP IgE-BF, specific IgG4 and IgA in DP SCIT treated patients $(n=12)$. The results were presented in 3 phases: initial: the original level; dummy is the phase treated with dummy beads depletion; and Pro-G is the phase treated with Pro$\mathrm{G}$ beads depletion. DP IgE-BF (a); Specific IgG4 and IgA, there are 4 subfigures: DP IgG4, Der p 1 IgG4, Der p 2 IgG4, and Der p 2

p 2 were removed after treatment with dummy beads (dummy), suggesting nonspecific depletion. When the sera were treated with Pro-G beads, about $80 \%$ of the IgG4 was depleted (Fig. 6b). For IgA, about $30 \%$ of Der $\mathrm{p}$ 2 -specific IgA was removed after dummy beads depletion
IgA (b). The result is presented as ratio to initial, so the initial level was set to 1 , the dummy level is the ratio of dummy/initial, and the Pro-G level is the ratio of Pro-G/initial. The horizontal line in each subfigure indicates the median level of before and after depletion. SCIT, subcutaneous immunotherapy; DP, Dermatophagoides pteronyssinus; Pro-G, Protein G.

(Fig. 6b). When treated with Pro-G beads (Pro-G), the effect is only slightly more than dummy beads depletion with a $40 \%$ IgA loss on average. However, the Pro-G depletion effect on IgA was very diverse suggesting a random effect. 


\section{Discussion/Conclusion}

In this open control study, both CSMS and QoL significantly improved after DP-SCIT treatment, confirming the efficacy of DP-SCIT [4]. The treatment induced a strong increase in DP-specific IgE-BF, indicating a strong IgE blocking activity. Moreover, even though the SCIT treatment was performed with a DP HDM extract, it also induced IgE blocking activity against the DF mite. The kinetics of DF IgE-BF induction is almost identical to DP IgE-BF and the 2 readouts are strongly correlated at all timepoints due to the extensive cross-reactivities toward the 2 mite species [20]. In China, both DP and DF mites are found in most households [21] and the vast majority of patients are IgE positive to both mites [3] as seen in the current study. Thus, even though patients were sensitized and most likely exposed to both DP and DF, the CSMS (overall reaction to the exposure) was significantly reduced suggesting that DP-SCIT treatment affects allergy to both mite species as a result of the immunological cross-reactivity.

Substantial increases in serum concentration of specific IgG4 against DP extract and Der $\mathrm{p} 1$ and Der $\mathrm{p} 2$ were induced by DP-SCIT in line with the previous studies [10, 22]. Moreover, the increases in allergen-specific IgG4 were also detectable in saliva and the concentrations correlated significantly with serum IgG4 measurements. IgG4 was present in serum before IgG4 with the same specificity appeared in saliva suggesting that it originated from serum and was transferred to saliva presumably by passive diffusion $[23,24]$. Thus, salivary IgG4 could be an alternative marker of the immunological effect of DPSCIT beyond 1 year of treatment.

Previous studies $[11,25]$ show that DP IgE-BF correlated with DP-specific IgG4 during DP-SCIT treatment and this was confirmed by the current study. In addition, DP IgE-BF also correlated with DP major allergen (Der $p$ 1 and Der $\mathrm{p} 2$ ) specific IgG4. It is noteworthy that the correlation became substantially stronger after DP-SCIT treatment reaching Pearson $r$ values of $>0.5$ for IgG4 toward DP, Der p 1, and Der p 2. This suggests that the effect of DP SCIT on IgG4 and IgE-BF is connected and that modulation of the response to the major allergens is key to the treatment effect. However, the correlations coefficients are only moderate indicating that other isotypes or serum components contribute to the blocking activity.

Apart from IgG4, IgA is also recognized as a potential IgE blocking antibody [26]. However, this study suggests that allergen-specific IgA probably only plays a minor or no role in the IgE-blocking activity because no correla- tion between serum Der p 2-specific IgA levels and IgE$\mathrm{BF}$ was observed. Moreover, no difference was observed between the DP-SCIT and the control group in Der $\mathrm{p}$ 2 -specific IgA1 and IgA2 in neither serum nor saliva at any treatment timepoint. Finally, Der p 2-specific serum IgA1 was unchanged and the serum concentrations of Der $\mathrm{p} 2$-specific IgA2 induced during treatment were very low further indicating a limited effect.

The functionality of the treatment-induced antibodies was further investigated by depletion of IgG4 in 12 sera obtained after 1 year of AIT. The depletion resulted in a consistent reduction of IgG4 to $25 \%$ of pretreatment levels and undetectable IgE-BF activity in $8 / 12$ of the depleted samples. Some depletion (30-50\%) of the low IgA serum concentration was observed for a part of the sera, and almost complete IgA depletion for $2 / 12$ sera. The removal of IgE-BF activity along with the consistent depletion of IgG4 support that primarily IgG4 is responsible for the blocking effect observed after DP-SCIT. A contribution from IgA cannot be completely ruled out on basis of the depletion experiments. However, the lack of a change in IgA1 and the low levels of IgA2 induced by treatment along with the removal of IgE-BF activity in sera only depleted for $20-40 \%$ of their IgA indicate that the contribution from IgA to the IgE-BF activity is minimal.

The role of IgA in immunotherapy remains controversial. The study of Jutel et al. [27] suggested that Der $p$ 1-specific T cells induced both TGF-beta and Der $\mathrm{p} 1$-specific IgA after immunotherapy. The study of Pilette et al. [28] also showed that $\mathrm{Phl} \mathrm{p}$ 5-specific IgA2 in serum increased significantly after 2 years of grass pollen SCIT with no change in the placebo group, suggesting that specific IgA2 is induced by grass pollen SCIT. However, when the IgE blocking activity was investigated the IgA containing serum fraction did not induce FAB inhibition, whereas the IgG4 containing fraction did. In line with this, the current study also demonstrated a small but significant increase in Der $\mathrm{p}$ 2-specific IgA2 in serum after SCIT; however, it was not statistically or functionally linked to IgE-BF possibly as a result of differences in epitope specificity for IgE/IgG4 compared to IgA [29]. Finally, the lack of correlation for Der p 2-specific IgA 1 and IgA2 between saliva and serum may suggest that the specific IgA $1 / \operatorname{IgA} 2$ in saliva and serum originate through different independent mechanisms as suggested by Meiler et al. [30], in contrast to IgG4. This should be investigated in a future study.

This study showed that the IgE blocking antibodies induced by DP-SCIT is not allergen-specific IgA but primary IgG4. In addition, DP IgE blocking activity is main- 
ly the result of IgG4 specific to DP major allergens. Moreover, DP-SCIT induced IgE blocking activity to DF as well as DP. DP-SCIT induced allergen-specific IgG4 seems to be transferred from serum to saliva and salivary allergen-specific IgG4 may constitute a noninvasive alternative marker to serum IgG4 beyond 1 year of treatment.

DP-SCIT induced a small but significant increase in Der p 2-specific IgA2 and future studies should further investigate the functionality of IgA in allergy and immunotherapy. In addition, future studies may also address the development of assays for Der p 1-specific IgA1 and IgA2 and their link to IgG4, IgE-BF, and maybe also clinical effect.

\section{Acknowledgements}

The authors would like to thank ALK colleagues Lars Harder Christensen for providing the $\mathrm{r}$ Der $\mathrm{p} 2$ and Der $\mathrm{p} 2 \mathrm{r}$ IgA assay standard reagent; Niels Johansen for the discussion during IgA assay development; Henrik Ipsen for essential statistical consultation and result discussion.

\section{Statement of Ethics}

The study was approved by the local Ethical Committees of each site, and informed consent was obtained from parent or guardian of every individual participant in the study. The study was registered on China Clinical Trial Registry with the registration ID of "ChiCTR-OIN-16009909." The study complied with the guidelines for human studies and was conducted ethically in accordance with the World Medical Association Declaration of Helsinki.

\section{Conflict of Interest Statement}

H.Z., H.C., P.A.W., J.N.L., and X.L. are the employee of ALK A/S, P.A.W., and J.N.L. own stocks in ALK A/S.

\section{Author Contributions}

Y.H., J.L., D.Z., S.Z., G.H., and Y.S. design the study, and recruit the patients and responsible for clinical management of patients and contribute equally in this study. H.Z. and H.C. perform the immunological measurements and data collection. X.L. performs data analysis and drafted the manuscript. P.A.W. and J.N.L. critically revised the manuscript, and all the authors approved the final version.

\section{References}

1 Jutel M, Akdis CA. Immunological mechanisms of allergen-specific immunotherapy. Allergy. 2011;66:725-32.

2 Głobińska A, Boonpiyathad T, Satitsuksanoa P, Kleuskens M, van de Veen W, Sokolowska $\mathrm{M}$, et al. Mechanisms of allergen-specific immunotherapy: diverse mechanisms of immune tolerance to allergens. Ann Allergy Asthma Immunol. 2018;121:306-12.

3 China Alliance of Research on Respiratory Allergic Disease; Li J, Sun B, Huang Y, Lin X, Zhao D, Tan G, et al. A multicentre study assessing the prevalence of sensitizations in patients with asthma and/or rhinitis in China. Allergy. 2009;64:1083-92.

4 Wang $\mathrm{H}$, Lin X, Hao C, Zhang C, Sun B, Zheng J, et al. A double-blind, placebo-controlled study of house dust mite immunotherapy in Chinese asthmatic patients. Allergy. 2006;61:191-7.

5 Karakoc-Aydiner E, Eifan AO, Baris S, Gunay E, Akturk E, Akkoc T, et al. Long-term effect of sublingual and subcutaneous immunotherapy in dust mite-allergic children with asthma/rhinitis: a 3-year prospective randomized controlled trial. J Investig Allergol Clin Immunol. 2015;25:334-42. Published correction appears in J Investig Allergol Clin Immunol. 2016;26(2):144.
6 Huang Y, Wang C, Cao F, Zhao Y, Lou H, Zhang L. Comparison of long-term efficacy of subcutaneous immunotherapy in pediatric and adult patients with allergic rhinitis. $\mathrm{Al}$ lergy Asthma Immunol Res. 2019;11:68-78.

7 Song Y, Long J, Wang T, Xie J, Wang M, Tan G. Long-term efficacy of standardised specific subcutaneous immunotherapy in children with persistent allergic rhinitis due to multiple allergens including house dust mites. J Laryngol Otol. 2018;132:230-5.

8 Aalberse RC, Stapel SO, Schuurman J, Rispens T. Immunoglobulin G4: an odd antibody. Clin Exp Allergy. 2009;39:469-77.

9 Shamji MH, Durham SR. Mechanisms of allergen immunotherapy for inhaled allergens and predictive biomarkers. J Allergy Clin Immunol. 2017;140:1485-98.

10 Lai X, Li J, Xiao X, Liu E, Zhang C, Wang H, et al. Specific IgG4 production during house dust mite immunotherapy among age, gender and allergic disease populations. Int Arch Allergy Immunol. 2013;160:37-46.

11 Zhao D, Lai X, Tian M, Jiang Y, Zheng Y, Gjesing B, et al. The functional IgE-blocking factor induced by allergen-specific immunotherapy correlates with IgG4 antibodies and a decrease of symptoms in house dust mite-allergic children. Int Arch Allergy Immunol. 2016;169:113-20.
12 Carlier FM, Sibille Y, Pilette C. The epithelial barrier and immunoglobulin A system in allergy. Clin Exp Allergy. 2016;46:1372-88.

13 Miranda DO, Silva DA, Fernandes JF, Queirós MG, Chiba HF, Ynoue LH, et al. Serum and salivary IgE, IgA, and IgG4 antibodies to Dermatophagoides pteronyssinus and its major allergens, Der p1 and Der p2, in allergic and nonallergic children. Clin Dev Immunol. 2011;2011:302739.

14 Liu Y, Xing Z, Wang J, Geng C. Salivary immunoglobulin A, E, and G4 levels specific to dermatophagoides pteronyssinus in allergic rhinitis patients treated with subcutaneous immunotherapy. Am J Rhinol Allergy. 2018; 32:458-64.

15 Canonica GW, Baena-Cagnani CE, Bousquet J, Bousquet PJ, Lockey RF, Malling HJ, et al. Recommendations for standardization of clinical trials with Allergen Specific Immunotherapy for respiratory allergy. A statement of a World Allergy Organization (WAO) taskforce. Allergy. 2007;62:317-24.

16 Juniper EF, Thompson AK, Ferrie PJ, Roberts JN. Validation of the standardized version of the rhinoconjunctivitis quality of life questionnaire. J Allergy Clin Immunol. 1999;104: 364-9. 
17 Juniper EF, Guyatt GH, Epstein RS, Ferrie PJ, Jaeschke R, Hiller TK. Evaluation of impairment of health related quality of life in asthma: development of a questionnaire for use in clinical trials. Thorax. 1992;47:76-83.

18 Petersen AB, Gudmann P, Milvang-Grønager P, Mørkeberg R, Bøgestrand S, Linneberg A, et al. Performance evaluation of a specific IgE assay developed for the ADVIA centaur immunoassay system. Clin Biochem. 2004;37: 882-92.

19 Christensen LH, Riise E, Bang L, Zhang C, Lund $\mathrm{K}$. Isoallergen variations contribute to the overall complexity of effector cell degranulation: effect mediated through differentiated IgE affinity. J Immunol. 2010;184:4966-72.

20 Thomas WR, Smith WA, Hales BJ, Mills KL, O'Brien RM. Characterization and immunobiology of house dust mite allergens. Int Arch Allergy Immunol. 2002;129:1-18.
21 Zheng YW, Lai XX, Zhao DY, Zhang CQ, Chen JJ, Zhang L, et al. Indoor allergen levels and household distributions in nine cities across China. Biomed Environ Sci. 2015;28: 709-17.

22 Chen J, Zhou Y, Wang Y, Zheng Y, Lai X, Westermann-Clark E, et al. Specific immunoglobulin $\mathrm{E}$ and immunoglobulin G4 toward major allergens of house-dust mite during allergen-specific immunotherapy. Am J Rhinol Allergy. 2017;31:156-60.

23 Vining RF, McGinley RA, Symons RG. Hormones in saliva: mode of entry and consequent implications for clinical interpretation. Clin Chem. 1983;29:1752-6.

24 Mostov KE. Transepithelial transport of immunoglobulins. Annu Rev Immunol. 1994; 12:63-84.

25 Feng M, Su Q, Lai X, Xian M, Shi X, Wurtzen $\mathrm{PA}$, et al. Functional and immunoreactive levels of IgG4 correlate with clinical responses during the maintenance phase of house dust mite immunotherapy. J Immunol. 2018;200: 3897-904.
26 Jiménez-Saiz R, Patil SU. The multifaceted B cell response in allergen immunotherapy. Curr Allergy Asthma Rep. 2018;18:66.

27 Jutel M, Akdis M, Budak F, Aebischer-Casaulta C, Wrzyszcz M, Blaser K, et al. IL-10 and TGF-beta cooperate in the regulatory $\mathrm{T}$ cell response to mucosal allergens in normal immunity and specific immunotherapy. Eur J Immunol. 2003;33:1205-14.

28 Pilette C, Nouri-Aria KT, Jacobson MR, Wilcock LK, Detry B, Walker SM, et al. Grass pollen immunotherapy induces an allergen-specific IgA2 antibody response associated with mucosal TGF-beta expression. J Immunol. 2007;178:4658-66.

29 Dodev TS, Bowen H, Shamji MH, Bax HJ, Beavil AJ, McDonnell JM, et al. Inhibition of allergen-dependent IgE activity by antibodies of the same specificity but different class. Allergy. 2015;70:720-4.

30 Meiler F, Klunker S, Zimmermann M, Akdis CA, Akdis M. Distinct regulation of IgE, IgG4 and IgA by $\mathrm{T}$ regulatory cells and toll-like receptors. Allergy. 2008;63:1455-63. 